\title{
Moving Object Classification Using Cloud Environment Through Haar Classifier
}

\author{
Mr.T.Mahalingam ${ }^{1}$,Dr.Shunmuganathan ${ }^{2}$,Miss.R.Jeevitha ${ }^{3}$. \\ Ph.D.Scholar ${ }^{1}$, Professor ${ }^{2}$,AsstiantProfessor ${ }^{3}$. \\ Sathyabama University ${ }^{1}$, R.M.K Engineering College ${ }^{2}$, Bhajarang Engineering College ${ }^{3}$
}

\begin{abstract}
In this paper we implement a moving Object classification system with cloud environment which is used for tracking any objects in non-ideal environment using android mobile. The tracked image is send to the cloud for storage and classification. The image is then retrieved by the system from the cloud storage (image sent by the android mobile) and it is classified based on the HAAR classifier algorithm. The HAAR classifier algorithm classifies the objects by comparing it with the objects in the cloud database and tracked objects and stores the result objects in the cloud for providing the details to be accessed anytime, anywhere from the remote system. A software approach for real time implementation of moving object classification is done through open source framework.
\end{abstract}

\section{Introduction}

Object tracking is an important task within the field of computer vision. The proliferation of highpowered computers, the availability of high quality and inexpensive video cameras, and the increasing need for automated video analysis has generated a great deal of interest in object tracking algorithms. Haar-like features are digital image features used in object recognition. The Haar classifier tends to be the most used method and with better success rate for face detection. A publication by Papageorgiou etal discussed working with an alternate feature set based on Haar wavelets instead of the usual image intensities. Viola and Jones adapted the idea of using Haar wavelets and developed the so-called Haar-like features.

Classification of the objects is a tedious process. In this project we are using android mobile phone for capturing the objects and the objects are then stored on the basis of their pixel value in the cloud environment(for example: if the pixel values is less than 10 or less than 20 or any specific range they are stored with their category and compared within that range avoiding work load for easier comparison) for reducing the effort of the algorithm so that it need not have to check or compare all the data's available in the database. This technique reduces the effort to half.cloud is used for the storage of the captured objects. by using the training datasets of the haar featuers the object has been classified.

The major task in this paper is to process the image at the real time to get the results. in recent times the cloud is the best storage for online application. The image which is stored in the cloud is first applied the mask to recoganize the object. Then they are separated according to their threshold pixel values and matched with the objects in the database and produces the details.

Related works are discussed in Section II and III describes the capturing and uploading objects. Section IV describes about Haar classification algorithm and training datasets in cloud database.

\section{Related Works}

The previous work In-Vehicle Infotainment (IVI) systems is an expanding field in the automobile industry. Cars from the BMW Group released in ECE/US have the feature of letting the user connect a mobile device to the head unit of the car, interweaving the mobile device with the vehicle. The BMW IVI system is soon to be released to the Chinese market (summer 2012), supporting widely used Chinese mobile device applications. Such mobile-to-car interweaving allows on the one hand the user to interact with their mobile phone through the larger display of the IVI system, accessing telephony, internet services, news, music, navigation, etc. and on the other hand allows the mobile to access virtually any data from the car head unitand system busses.

The aim of this project was to develop an object recognition module to an iPhone AR prototype application for the BMW Connected Drive Lab, located in Shanghai China. The ambition was to implement this prototype so that it could be used as a basis for an interactive diagnostics tool, handbook or similar, allowing further information about the identified objects and graphics to be layered on screen. The application, on prototype level, is a stand-alone tool not dependent on connection or communication with the existing IVIsystem. Future versions of this application could be integrated with the IVI system and present diagnostic data on the mobile device. Such an AR module could be useful in a number of tools accompanying a car, e g. using the 
mobile device as a diagnostic tool (to check oil, washer uid, tire pressure etc.), as an interactive car handbook or as a remote control towards the IVI system.

The project was divided into two parts, the rst focused on object recognition and the second focused on the user interface interaction and graphical overlay onto the camera-provided images. The first part is presented in the current bachelor thesis, and the second part is covered by the bachelor thesis of Gabriel Tholsgard, with whom collaboration have been extensive. The current report describes the work of implementing image processing and applying object recognition to parts on the car dashboard provided in a video stream, and choosing an efficient approach that takes the relevant invariance properties in to account.

\section{Existing system}

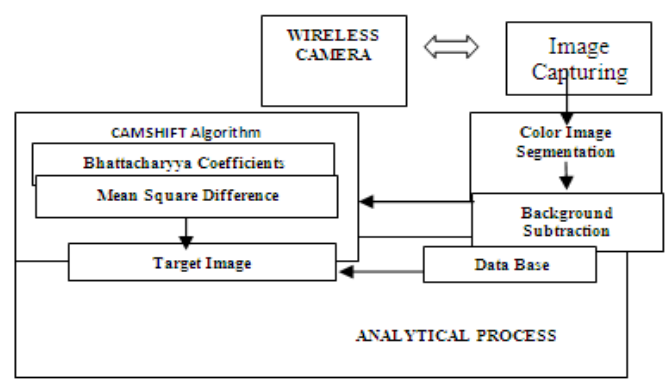

System Model

In the existing sytem the objects are captured using wireless camera, captured image is segmented by using colorimage segmentation then it is background subtracted the moving object is tracked the centroid of the moving object is find out by the means square difference and Bhattacharyya Coefficients. Then the target image is obtained by comparing with the result from camshift algorithm with the database objects.

In the existing system the object are stored in the database which are difficult to be accessed by the remote system. In the proposed system the cloud storage is used so that the objects can be accessed any where, anytime remotely whereas in the previously used system we cannot access it remotely.

\section{Proposed System}

1. Take current image from android mobile phone and upload in cloud.

2. Take difference between them similar cached previous image and current image by using HAAR Clasifier.

3. Create or Select Thresholding limit .

4. Difference of image is greater then threshold objectis detected

5. Find centroid of detected object

6. Generate template and take coordinate of template

7. (Template matching algorithm)IF the template matching is successfulTHENIF the tracker has NOT detected motion ofthe objectAND the detector hasTHEN goto STEP 1 (get a newtemplate)ELSE goto STEP 5 (get the x, yposition)ELSE goto STEP 1 (get a new template )

8. Obtain the position $\mathrm{P}(\mathrm{x}, \mathrm{y})$ of the match and pass iton to the pan-tilt automation module for analysis.

9. Get the direction of horizontal and verticalmovement of tracked object.

10. On based of movement within certain dimension itdecide movement of camera in clockwise or anticlockwise

11. Else go to step 1.

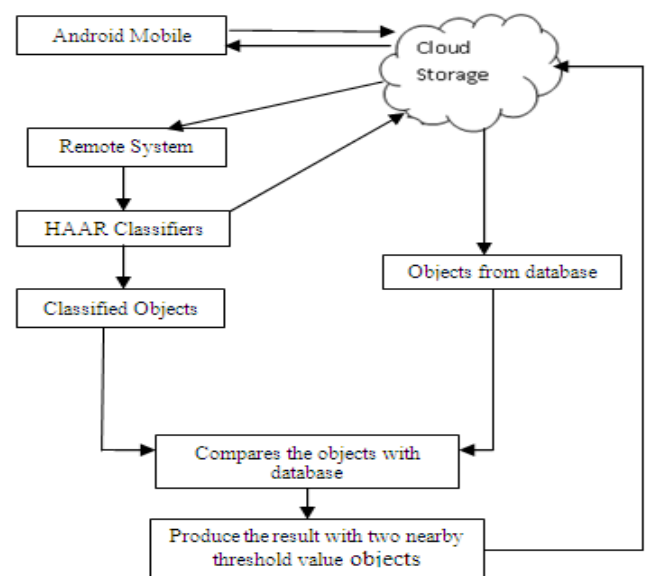


Flow chart

Here the objects are tracked using the android mobile and the captured objects are stored in the cloud database. The cloud database contains the objects with Conclusion.

The object to be tracked can also be identified using HAAR classifier through machine learning. Information exchange between the classification module and the tracking module also enhances their adaptation to real complex occlusion scenes. This method achieves higher classification accuracy in tracking performance in comparison with wired camera. The templates used for the matching purposes are generated dynamically. This ensures that any change in the pose of the object does not hinder the tracking procedure. By processing real-time images and communicating wirelessly in outdoor environments, we can track moving objects against complex, cluttered backgrounds.

\section{Conclusion And Future Work}

To make the system an adaptive tracking process we intend to build an embedded system for automating the tracking process the camera is mounted on a pan tilt mechanism coupled with stepper motor, which is synchronized with a tracking algorithm. As and when the object being tracked moves out of the viewing range of the camera, the setup is automatically adjusted to move the camera so as to keep the object of about 360 degree field of view. The system is capable of handling entry and exit of an object in existing sytem. The performance of the proposed Object tracking system can been demonstrated in real time environment both for indoor and outdoor by including a variety of disturbances and a means to detect a loss of track. At the same time the object to be tracked can be classified using HAAR Classifier. Further work is currently underway to extend for tracking multiple objects at same time by enhancing the Bhattacharyya coefficients and also the major future work data storage cloud environment must be adapted to hadoop in cloud based environment.

\section{References}

[1] D. Comaniciu and V. Ramesh, Real Time Tracking of Non-Rigid Objects using Mean Shift, Computer vision and Pattern Recognition, 2000 proceedings IEEE conference.

[2] OpenCV Library, Intel Corporation.

[3] D.Marshall, Region Growing, Vision Systems, 1994.

[4] E.Pauwles and G.Frederix. Non-Parametric Clustering for Image Segmentation and Grouping. Computer Vision and Image Understanding, 75 nos. 1/2, pp. 73-85, 1999.

[5] Y.Fang and T.Tan, A Novel Adaptive Colour Segmentation Algorithm and its Application to Skin Detection. In Proc.BMVC 2000, pp.23-31. BMVA, 2000.

[6] G.D.Finlayson and G.Y.Tian. Colour Normalisation for Colour Object Recognition. International Journal of Pattern Recognition and Artificial Intelligence, pp. 1271-1285, 1999.

[7] W.Skarbek and A.Koschan. Colour Image Segmentation : A Survey. Technischer Bericht 94-32, Technical University of Berlin, 1994.

[8] T.Gevers, S. Ghebreab, and A.W.M. Smeulders. Colour Invariant Snakes. In Proc. BMVC 1998, pp. 578-588. BMVA, 1998.

[9] G.J.Klinker, A. Shafer, and T. Kanada. A Physical Approach to Colour Image Understanding. International Journal of Computer Vision, 4, pp. 7-38, 1990.

[10] N.A. Thacker, I. Abraham and P. Courtney. Supervised Learning Extensions to the CLAM Network. Neural Networks, 10 no. 2, pp 315-326, 1997.

[11] G. Wyszecki and W.S. Stiles. Color Science: Concepts and Methods, Quantitative Data and Formulae (2nd Edition) John Wiley and Sons, New York, 1982.

[12] P.A. Bromiley, P. Courtney and N.A. Thacker. A Case Study in the use of ROC Curves for Algorithm Design. In Proc. BMVC 2001, BMVA, 2001.

[13] G. R. Bradski and J. Davis, Motion Segmentation and Pose Recognition with Motion History Gradients, Machine.

[14] D. Comaniciu, V. Ramesh, P. Meer, "Kernel-Based Object Tracking”, IEEE Trans. on Pattern Analysis and Machine Intelligence, vol.25, No. 5, 2003.

About Authors:

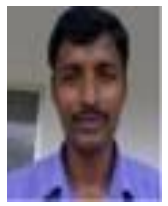

MahaLingam .T obtained Masters Degree in BharahadasanUniveristy,pursuing $\mathrm{Ph} . \mathrm{d}$ in Satyabama University and working as Assistant professor in Bhajarang Engineering College.

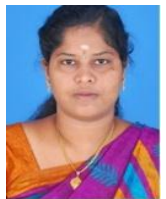

Jeevitha.R, obtained Masters Degree in Sri Sai Ram Engineeering College,(Anna University Rank Holder) working as Assistant Professor in Bhajarang Engineering College. 